\title{
An Interior a Priori Estimate for Parabolic Difference Operators and an Application
}

\author{
By Magnus Bondesson
}

\begin{abstract}
A general class of finite-difference approximations to a parabolic system of differential equations in a bounded domain $\Omega$ is considered. It is shown that if a solution $U_{h}$ of the discrete problem converges in a discrete $L^{2}$ norm to a solution $U$ of the continuous problem as the mesh size $h$ tends to zero, then the difference quotients of $U_{h}$ converge to the corresponding derivatives of $U$, the convergence being uniform on any compact subset of $\Omega$. In particular, $U_{h}$ converges uniformly on compact subsets to $U$ as $h$ tends to zero, provided there is convergence in the discrete $L^{2}$ norm. The main part of the paper is devoted to the establishment of an a priori estimate for the solutions of the discrete problem. This estimate is then used to derive the stated result.
\end{abstract}

1. Introduction. Let $\Omega$ be a bounded domain in $R^{1+d}$. The points in $R^{1+d}$ will be denoted by $X=(t, x)=\left(t, x_{1}, \cdots, x_{d}\right)$. In $\Omega$ we consider the inhomogeneous system of differential equations

$$
L U \equiv D_{t} U-\sum_{|\alpha| \leqq M} A_{\alpha} D_{x}^{\alpha} U=F,
$$

where the $N$-vector $F(t, x)$ and the $N \times N$ matrices $A_{\alpha}(t, x)$ are in $C^{\infty}(\Omega)$. We also assume that the differential operator $L$ is parabolic in $\Omega$ in Petrovskiri's sense, which implies that any solution of $(1.1)$ is in $C^{\infty}(\Omega)$.

Let $\lambda$ be a positive constant and let $h$ and $k$ be positive parameters such that $k=\lambda h^{M}$. We introduce the mesh

$$
\left\{X \in R^{1+d}: X=\left(\nu_{0} k, \nu_{1} h, \cdots, \nu_{d} h\right), \nu_{i} \text { integers }\right\},
$$

and denote by $\Omega_{h}$ the set of meshpoints in $\Omega$. Let the difference operator $L_{h}$ be consistent with the differential operator $L$ and be determined by

$$
L_{h} U(t, x)=k^{-1} \sum_{\nu}\left[a_{\nu}(t, x, h) U(t, x+\nu h)-b_{\nu}(t, x, h) U(t-k, x+\nu h)\right],
$$

where $a$, and $b$, are smooth $N \times N$ matrix valued functions. Furthermore, we require that $L_{h}$ is parabolic in $\Omega$ in the sense of F. John. Then we approximate (1.1) by the equation

$$
L_{h} U_{h}=M_{h} F, \quad X \in \Omega_{h}^{o},
$$

where $M_{h}$ is an operator consistent with the identity operator and $\Omega_{h}^{o}$ is, for instance, the maximal subset of $\Omega_{h}$ in which $L_{h} U$ and $M_{h} U$ are determined by the values of $U$ in $\Omega$.

Received May 15, 1970.

AMS 1970 subject classifications. Primary 65N10, 65N15.

Key words and phrases. Parabolic difference operator, a priori estimate, boundary value problem, convergence in maximum norm, convergence of difference quotients.

Copyright $\odot$ 1971, American Mathematical Society 
Let $U$ be a solution of (1.1) and $U_{h}$ be a solution of (1.2). Further, let $\Omega_{1} \subset \subset$ $\Omega_{2} \subset \subset \Omega$ and set $\Omega_{i}^{T}=\Omega_{i} \cap\{X: t \leqq T\}, i=1,2$. Our result is that if (1.2) approximates (1.1) with order of accuracy $p$ and the same holds for the difference operator $Q_{h}$ and the differential operator $Q$, then

$$
\left|Q_{h} U_{h}-Q U\right|_{h, \Omega_{1}} T \leqq C\left(h^{p}+\left\|U_{h}-U\right\|_{h, \Omega_{2}} T\right), \quad h \leqq h_{0}, \quad T \in R,
$$

where the constants $C$ and $h_{0}$ are independent of the particular solution $U_{h}$ and

$$
\begin{aligned}
|V|_{h, \Omega} & =\max _{X \in \Omega_{h}}|V(X)|, \\
\|V\|_{h, \Omega} & =\left(k h^{d} \sum_{X \in \Omega_{h}}|V(X)|^{2}\right)^{1 / 2} .
\end{aligned}
$$

From (1.3) we conclude that if $U$ is a solution of a mixed problem for (1.1) and $U_{h}$ is a solution of a corresponding discrete problem with $L_{h} U_{h}=M_{h} F$ in $\Omega_{h}^{\circ}$ and such that $\left\|U_{h}-U\right\|_{h, \Omega_{2}} \rightarrow 0$ as $h \rightarrow 0$, then $Q_{h} U_{h}$ converges uniformly in $\Omega_{1}$ to $Q U$.

To derive (1.3), we start by proving, in Section 2, three lemmas, which are consequences of our assumptions about the operator $L_{h}$. In Section 3 , we use these lemmas to prove an a priori inequality for the difference quotients of functions $V$ defined in the meshpoints. This inequality essentially states that

$$
\left\|\partial_{x}^{\alpha} V\right\|_{h, \Omega_{1} T} \leqq C\left(\left\|L_{h} V\right\|_{h_{, \Omega_{2}} T}+\|V\|_{h_{\Omega_{2}} T}\right), \quad h \leqq h_{0}, \quad|\alpha| \leqq M, \quad T \in R .
$$

In Section 4 , we combine the a priori inequality with the discrete Sobolev inequality to obtain (1.3). Finally, in Section 5, we illustrate our results by two simple examples.

The methods used in Section 3 are to a great extent taken from [11], where results similar to ours were proved for the elliptic case. Related questions for the pure initial-value problem for the homogeneous counterparts of (1.1) and (1.2) have been considered by Thomée [10] and Widlund [12]. In this case, convergence of the discrete solution $U_{h}$ to the exact solution $U$ is not assumed, but is part of the result.

I wish to thank Professor Vidar Thomée for suggesting the problem and for his constant encouragement during the writing of this paper.

2. Notation and Preliminaries. Let $\Omega$ be a bounded domain in $R^{1+d}$. The points in $R^{1+d}$ will be written $X=(t, x)$ with $x \in R^{d}$. If $\Omega_{1}$ is a domain such that $\bar{\Omega}_{1} \subset \Omega$, we shall write $\Omega_{1} \subset \subset \Omega$. Unless otherwise stated, all the functions considered have their values in the $N$-dimensional complex vector space. For $N$-vectors $V=\left(v_{1}\right.$, $\left.\cdots, v_{N}\right)$ we use the Euclidean norm $|V|=\left(\sum\left|v_{i}\right|^{2}\right)^{1 / 2}$ and for $N \times N$ matrices $A$ the induced norm $|A|=\sup \{|A V|:|V|=1\}$. If $A$ is an $N \times N$ matrix, we let $\lambda_{j}(A), j=1, \cdots, N$, denote the eigenvalues and $\rho(A)$ denote the spectral radius, i.e. $\max \left|\lambda_{i}(A)\right|$.

Greek letters $\alpha, \beta, \gamma, \mu, \nu$, will denote $d$-dimensional multi-indices with integral components. If $\alpha=\left(\alpha_{1}, \cdots, \alpha_{d}\right)$ is such an index, we shall write $|\alpha|=\sum\left|\alpha_{i}\right|$. We define the translation operators $T_{t}^{i} T_{x}^{\alpha}$ by

$$
T_{t}^{i} T_{x}^{\alpha} U(t, x)=U(t+j k, x+\alpha h)
$$

and the difference operators $\partial_{t}, \bar{\partial}_{t}$, and $\partial_{s}$ by

$$
\partial_{t}=\frac{1}{k}\left(T_{t}-I\right), \quad \bar{\partial}_{t}=T_{t}^{-1} \partial_{t}, \quad \partial_{x i}=\frac{1}{h}\left(T_{x}^{e i}-I\right),
$$


where $I$ is the identity operator and $e_{i}$ is the $i$ th unit vector in $R^{d}$. If the integer $j$ and the $\alpha_{i}$ 's are nonnegative, we will use the notation

$$
D_{t}^{j} D_{x}^{\alpha}=\frac{\partial^{j+|\alpha|}}{\partial_{t}^{j} \partial_{x_{1}}^{\alpha_{1}} \cdots \partial_{x_{d}}^{\alpha_{d}}}, \quad \partial_{t}^{j} \partial_{x}^{\alpha}=\partial_{t}^{j} \partial_{x_{1}}^{\alpha_{1}} \cdots \partial_{x_{d}}^{\alpha_{d}} .
$$

Note that these definitions depend on the positive numbers $k$ and $h$. However, we preferred not to indicate this in the notation. Also, we shall frequently suppress the subscripts $t$ and $x$.

Now, consider a differential operator $L$ defined by

$$
L U=\left(D_{t}-P\left(D_{x}\right)\right) U \equiv D_{t} U-\sum_{|\alpha| \leqq M} A_{\alpha} D_{x}^{\alpha} U
$$

where the $N \times N$ matrices $A_{\alpha}(t, x)$ are assumed to be in $C^{\infty}(\Omega)$. This operator is said to be parabolic in $\Omega$ in Petrovskir's sense if for any $X \in \Omega$ there is a positive constant $C$ such that

$$
\max _{j}\left(\operatorname{Re} \lambda_{i}\left(\sum_{|\alpha|=M} A_{\alpha}(X)(i \xi)^{\alpha}\right)\right) \leqq-C|\xi|^{M}, \quad \xi \in R^{d}, \quad \xi^{\alpha}=\xi_{1}^{\alpha_{1}} \cdots \xi_{d}^{\alpha_{d}} .
$$

Throughout this paper, we shall assume that the positive parameters $k$ and $h$ satisfy a relation $k=\lambda h^{M}, \lambda=$ constant. Let $C_{N}^{\infty}(\Omega, h)$ be the set of $N \times N$ matrix valued functions $A=A(X, h)$ such that for any $\Omega_{1} \subset \subset \Omega, A$ is infinitely differentiable in $\Omega_{1} \times\left[0, h_{0}\right]$ for some positive $h_{0}$. We then approximate the differential operator $L$ by a difference operator $L_{h}$ given by

$$
L_{h} U(t, x)=\frac{1}{k}\left[A_{h} U(t, x)-B_{h} T_{t}^{-1} U(t, x)\right],
$$

where the operators $A_{h}$ and $B_{h}$ have the same form, e.g.

$$
A_{h}=\sum_{|\nu| \leqq K} a_{\nu}(t, x, h) T_{x}^{\nu}, \quad a_{\nu} \in C_{N}^{\infty}(\Omega, h), \quad K<\infty .
$$

We say that $L_{h}$ is consistent with $L$ in $\Omega$ if for any $U \in C^{\infty}(\Omega)$ and any $X \in \Omega$

$$
L_{h} U(X)-L U(X)=o(1) \quad \text { as } h \rightarrow 0 .
$$

$L_{h}$ is said to be a consistent operator if it is consistent with some differential operator of the form (2.1). The notion of consistency is introduced analogously for other types of difference operators.

Our first lemma gives a simple representation of the operator $L_{h}$.

LEMMA 2.1. Suppose that $k=\lambda h^{M}$. Then the difference operator $L_{h}$ given by (2.2) is consistent with the differential operator $L$ in (2.1) if and only if $A_{h}$ is consistent with I, the identity operator, and

$$
L_{h}=A_{h} \bar{\partial}_{t}-P_{h},
$$

where $P_{h}$ has the form

$$
P_{h}=\sum_{|\alpha| \leqq M, \nu} c_{\alpha \nu}(t, x, h) T_{t}^{-1} T_{x}^{\nu} \partial_{x}^{\alpha}, \quad c_{\alpha \nu} \in C_{. v}^{\infty}(\Omega, h)
$$

with $c_{\alpha}, \neq 0$ only for a finite set of indices and

$$
\begin{aligned}
c_{\alpha \nu}(t, x, h) & =c_{\alpha \nu}(t, x, 0), \quad \alpha \neq 0, \\
\sum_{\nu} c_{\alpha \nu}(t, x, 0) & =A_{\alpha}(t, x) .
\end{aligned}
$$


Proof. The proof of the sufficiency part is obvious. In order to prove that the conditions are necessary we write

$$
L_{h}=A_{h} \bar{\partial}_{t}-\frac{1}{\lambda h^{M}}\left(B_{h}-A_{h}\right) T_{t}^{-1}=A_{h} \bar{\partial}_{t}-\tilde{P}_{h} T_{t}^{-1}=A_{h} \bar{\partial}_{t}-P_{h},
$$

where for certain $\tilde{p}_{v} \in C_{N}^{\infty}(\Omega, h)$

$$
\tilde{P}_{h}=h^{-M} \sum_{\nu} \tilde{p}_{\nu}(t, x, h) T_{x}^{\nu}
$$

Since $L_{h}$ is assumed to be consistent with $L$, we find by taking $U(t, x)=U(x) \in$ $C^{\infty}\left(R^{d}\right)$ that for $(t, x) \in \Omega$

$$
\left(\tilde{P}_{h}-P\left(D_{x}\right)\right) U(t, x)=o(1) \quad \text { as } h \rightarrow 0 .
$$

This means that, for fixed $t$, Lemma 2.2 in [11] applies, giving

$$
\tilde{P}_{h}=\sum_{|\alpha| \leqq M, \nu} c_{\alpha \nu}(t, x, h) T_{x}^{\nu} \partial_{x}^{\alpha},
$$

where the coefficients $c_{\alpha \nu}$ satisfy (2.3). The construction in that lemma shows that $c_{\alpha \nu} \in C_{N}^{\infty}(\Omega, h)$ and hence $P_{h}=\tilde{P}_{h} T_{t}^{-1}$ has the stated properties. From (2.3), we infer that $P_{h}$ is consistent with $P\left(D_{x}\right)$. Thus, we have for any $U \in C^{\infty}(\Omega)$ and any $(t, x) \in \Omega$

$$
A_{h} \bar{\partial}_{t} U(t, x)-D_{t} U(t, x)=o(1) \quad \text { as } h \rightarrow 0,
$$

and, by taking $U(t, x)=t U(x)$, we get $\sum_{\nu} a_{\nu}(t, x, 0)=I$, which implies the consistency of $A_{h}$ with $I$. This completes the proof of the lemma.

We now introduce the principal parts $A_{h}^{0}, B_{h}^{0}$, and $L_{h}^{0}$ of the operators $A_{h}, B_{h}$, and $L_{h}$, respectively, namely

$$
A_{h}^{0}=\sum_{\nu} a_{\nu}(t, x, 0) T_{x}^{\nu}, \quad L_{h}^{0}=\frac{1}{k}\left(A_{h}^{0}-B_{h}^{0} T_{t}^{-1}\right),
$$

where $B_{h}^{0}$ is defined in the same way as $A_{h}^{0}$. If $L_{h}$ is a consistent operator, we find from Lemma 2.1

$$
L_{h}^{0}=A_{h}^{0} \bar{\partial}_{t}-\sum_{|\alpha|=M, \nu} c_{\alpha \nu}(t, x, 0) T_{t}^{-1} T_{x}^{\nu} \partial_{x}^{\alpha} .
$$

Further, we introduce the symbols

$$
\begin{array}{cl}
A_{h}^{0}(\xi)=A_{h}^{0}(\xi, X)=\sum_{\nu} a_{\nu}(X, 0) \exp (i h \nu \cdot \xi), & \xi \in R^{d}, \quad \nu \cdot \xi=\sum_{i=1}^{d} \nu_{i} \xi_{i}, \\
L_{h}^{0}(\tau, \xi)=L_{h}^{0}(\tau, \xi, X)=\frac{1}{k}\left[A_{h}^{0}(\xi)-e^{-i k \tau} B_{h}^{0}(\xi)\right], \quad \tau \in R, \quad \xi \in R^{d},
\end{array}
$$

corresponding to the operators $A_{h}^{0}$ and $L_{h}^{0}$. Here, $B_{h}^{0}(\xi)$ is defined in the same way as $A_{h}^{0}(\xi)$.

We shall assume that for any $\Omega_{1} \subset \subset \Omega$ there is a positive constant $C$ such that

$$
\mid \text { det } A_{h}^{0}(\xi, X) \mid \geqq C, \quad X \in \Omega_{1} .
$$

Consequently, the following notation makes sense:

$$
E_{h}^{0}(\xi)=E_{h}^{0}(\xi, X)=A_{h}^{0}(\xi, X)^{-1} B_{h}^{0}(\xi, X) .
$$


Definition. The operator $L_{h}$ in (2.2) is said to be parabolic in $\Omega$ in the sense of F. John [4] if it is consistent and if for any $\Omega_{1} \subset \subset \Omega$ there is a positive constant $C$ such that $(2.5)$ is satisfied and

$$
\rho\left(E_{h}^{0}(\xi, X)\right) \leqq 1-C k|\xi|^{M}, \quad\left|h \xi_{i}\right| \leqq \pi, \quad X \in \Omega_{1}
$$

The next lemma will be fundamental for the a priori estimate in Section 3.

LEMMA 2.2. If $L_{h}$ is a parabolic difference operator in $\Omega$, there exists for any $\Omega_{1} \subset \subset \Omega$ a constant $C_{0}$ such that

$$
\left|L_{h}^{0}(\tau, \xi, X)^{-1}\right| \leqq C_{0}|\xi|^{-M}, \quad \xi \neq 0, \quad\left|h \xi_{j}\right| \leqq \pi, \quad X \in \Omega_{1} .
$$

Proof. We have $L_{h}^{0}(\tau, \xi)=(1 / k) A_{h}^{0}(\xi)\left(I-e^{-i k \tau} E_{h}^{0}(\xi)\right)$ and so it is enough to prove that $\left|\left(I-e^{-i k r} E_{h}^{0}(\xi)\right)^{-1}\right| \leqq C /\left(k|\xi|^{M}\right)$. But we know (Lemma 3.4 in [10]) that

$$
\left|\left(E_{h}^{0}(\xi)\right)^{n}\right| \leqq C_{1} \exp \left(-C_{2} n k|\xi|^{M}\right), \quad\left|h \xi_{i}\right| \leqq \pi,
$$

uniformly for $X \in \Omega_{1}$ and hence we get for $\xi \neq 0$

$$
\left|\left(I-e^{-i k r} E_{h}^{0}(\xi)\right)^{-1}\right| \leqq \sum_{n=0}^{\infty}\left|\left(E_{h}^{0}(\xi)\right)^{n}\right| \leqq C_{1} /\left(1-\exp \left(-C_{2} k|\xi|^{M}\right)\right) \leqq C /\left(k|\xi|^{M}\right),
$$

as required.

We will need some facts about Fourier series. The set of $N$-vector valued functions defined on the mesh $\left\{X \in R^{1+d}: X=(j k, \nu h)=\left(j k, \nu_{1} h, \cdots, \nu_{d} h\right), j, \nu_{i}\right.$ integers, $\left.k=\lambda h^{M}\right\}$ is denoted by $\mathfrak{T}_{h}$. In the subset $l_{k, h}^{2}=\left\{U \in \mathfrak{T}_{h}: \sum_{i, \nu}|U(j k, \nu h)|^{2}<\infty\right\}$ we use the norm

$$
\|U\|_{h}=\left(k h^{d} \sum_{i, \nu}|U(j k, \nu h)|^{2}\right)^{1 / 2} .
$$

For $U \in l_{k, h}^{2}$ we introduce the Fourier transform

$$
\hat{U}(\tau, \xi)=k h^{d} \sum_{i, \nu} U(j k, \nu h) \exp [-i(j k \tau+\nu \cdot h \xi)], \quad \tau \in R, \quad \xi \in R^{d},
$$

with the sum defined as a limit in $L^{2}(Q)$, where $Q=\left\{(\tau, \xi):|k \tau| \leqq \pi,\left|h \xi_{j}\right| \leqq \pi\right.$, $j=1, \cdots, d\}$. Conversely, if $\tilde{U} \in L^{2}(Q)$ the function $U$ defined by

$$
U(j k, \nu h)=(2 \pi)^{-1-d} \int_{Q} \tilde{U}(\tau, \xi) \exp [i(j k \tau+\nu \cdot h \xi)] d \tau d \xi
$$

belongs to $l_{k, h}^{2}$ and is the unique function in this space having the Fourier transform $\tilde{U}$. The Parseval relation holds, that is

$$
\|U\|_{h}^{2}=(2 \pi)^{1+d} \int_{Q}|\hat{U}(\tau, \xi)|^{2} d \tau d \xi
$$

We note that

$$
\left(\partial_{x}^{\alpha} U\right)^{\hat{(}}(\tau, \xi)=h^{-|\alpha|} \prod_{i=1}^{d}\left(e^{i h \xi_{i}}-1\right)^{\alpha_{i}} \hat{U}(\tau, \xi)=\partial_{x}^{\alpha}(\xi) \hat{U}(\tau, \xi) .
$$

We shall consider also the space $l_{h}^{2}$ of functions $U$ defined on the mesh $\left\{x \in R^{d}: x=\nu h\right\}$ and such that $\sum_{\nu}|U(\nu h)|^{2}<\infty$. In this space the counterparts of the facts above are obvious. 
Now let the principal part operator $L_{h}^{0}$ be parabolic and have coefficients which are independent of $X$. It then follows from above that $\left(L_{h}^{0} U\right)^{\wedge}(\tau, \xi)=L_{h}^{0}(\tau, \xi) \cup(\tau, \xi)$, and hence for $\xi \neq 0$ we have $\left(\partial_{x}^{\alpha} U\right)^{\wedge}(\tau, \xi)=\partial_{x}^{\alpha}(\xi) L_{h}^{0}(\tau, \xi)^{-1}\left(L_{h}^{0} U\right)^{\wedge}(\tau, \xi)$. This is the key to the following lemma, which gives a representation for $\partial_{x}^{\alpha} U(t, x),|\alpha|=M$.

LEMMa 2.3. Let $L_{h}^{0}$ be the principal part of a parabolic difference operator with coefficients independent of $(t, x)$ and let $T$ and $T_{0}$ be real numbers. Suppose that $U$ is a mesh-function such that $U(j k, \cdot) \in l_{h}^{2}$ for any integer $j$ and $U(j k, \cdot)=0$ for $j k \leqq T_{0}$. If $F=L_{h}^{0} U$ for $t \leqq T$ and $F=0$ otherwise, then for $|\alpha|=M$ and $t \leqq T$,

$$
\partial_{x}^{\alpha} U(t, x)=(2 \pi)^{-1-d} \int_{0} \partial_{x}^{\alpha}(\xi) L_{h}^{0}(\tau, \xi)^{-1} \hat{F}(\tau, \xi) \exp [i(t \tau+x \cdot \xi)] d \tau d \xi .
$$

Proof. It suffices to consider $T_{0}=0$. The condition (2.5) shows that $\left(A_{h}^{0}\right)^{-1}$ is a bounded operator on $l_{h}^{2}$. Hence the problem

$$
\begin{aligned}
L_{h}^{0} V & =\partial_{x}^{\alpha} F, & t & =j k>0, \\
V & =0, & t & =j k \leqq 0,
\end{aligned}
$$

has a unique solution $V$ in $\mathfrak{T}_{h}$ such that $V(j k, \cdot) \in l_{h}^{2}$. Since $L_{h}^{0}$ commutes with $\partial_{x}^{\alpha}$, we have $V(t, x)=\partial_{x}^{\alpha} U(t, x)$ for $t \leqq T$.

Let $|\alpha|=M$. For meshpoints $(t, x)$ we denote the right member in $(2.7)$ by $H(t, x)$. By Lemma 2.2, $\left|\partial_{x}^{\alpha}(\xi) L_{h}^{0}(\tau, \xi)^{-1}\right| \leqq$ constant, $\xi \neq 0$, and so $H \in l_{k, h}^{2}$ and consequently also $H(t, \cdot) \in l_{h}^{2}$. We claim that $H$ satisfies (2.8). This would imply the lemma. Taking Fourier transforms we see that $L_{h}^{0} H=\partial_{x}^{\alpha} F$ and hence it only remains to prove that $H(j k, x)=0$ for $j \leqq 0$. But

$$
H(t, x)=G * F(t, x)=k h^{d} \sum_{n, v} G(t-n k, x-\nu h) F(n k, \nu h),
$$

where the mesh-function $G$ has the Fourier transform $\hat{G}=\partial_{x}^{\alpha}(\xi) L_{h}^{0}(\tau, \xi)^{-1}$, and so it suffices to prove that $G(j k, x)=0$ for $j<0$. We have, for $j<0$,

$$
\begin{aligned}
(2 \pi)^{1+d} G(j k, x) & =k \int_{\left|h \xi_{i}\right| \leqq \pi}\left[\int_{|k \tau| \leqq \pi} e^{i j k \tau}\left(I-e^{-i k \tau} E_{h}^{0}(\xi)\right)^{-1} d \tau\right] \partial_{x}^{\alpha}(\xi) A_{h}^{0}(\xi)^{-1} e^{i x \cdot \xi} d \xi \\
& =\frac{1}{i} \int_{\left|h \xi_{i}\right| \leqq \pi}\left[\int_{|z|-1} z^{-1-i}\left(I-z E_{h}^{0}(\xi)\right)^{-1} d z\right] \partial_{x}^{\alpha}(\xi) A_{h}^{0}(\xi)^{-1} e^{i x \cdot \xi} d \xi \\
& =0,
\end{aligned}
$$

since the condition (2.6) shows that the function $\left(I-z E_{h}^{0}(\xi)\right)^{-1}, \xi \neq 0$, is analytic in $z$ for $|z| \leqq 1$ and the inner integral thus is equal to zero. The proof is complete.

We also state the Leibniz rule which will be used frequently in the technical calculations later on.

The Leibniz Rule. If $A$ is an $N \times N$ matrix valued function with rows in $\mathfrak{T}_{h}$ and if $U \in \mathfrak{M}_{h}$, we have

$$
\partial^{\bar{\alpha}}(A U)=\sum_{\bar{\beta} \leq \bar{\alpha}}\left(\begin{array}{l}
\bar{\alpha} \\
\bar{\beta}
\end{array}\right)\left(T^{\bar{\beta}} \partial^{\bar{\alpha}-\bar{\beta}} A\right)\left(\partial^{\bar{\beta}} U\right)=\sum_{\bar{\beta} \leq \bar{\alpha}}\left(\begin{array}{l}
\bar{\alpha} \\
\bar{\beta}
\end{array}\right)\left(\partial^{\bar{\beta}} A\right)\left(T^{\bar{\beta}} \partial^{\bar{\alpha}-\bar{\beta}} U\right),
$$

where

$$
\bar{\alpha}=\left(\alpha_{0}, \alpha\right), \quad T^{\bar{\alpha}}=T_{t}^{\alpha_{0}} T_{x}^{\alpha}, \quad \partial^{\bar{\alpha}}=\partial_{t}^{\alpha_{0}} \partial_{x}^{\alpha}, \quad\left(\begin{array}{c}
\bar{\alpha} \\
\bar{\beta}
\end{array}\right)=\prod_{i=0}^{d}\left(\begin{array}{c}
\alpha_{i} \\
\beta_{i}
\end{array}\right) .
$$


3. An Interior a Priori Estimate. For any set $\Omega$ in $R^{1+d}$ and any real number $T$, we put $\Omega^{T}=\{(t, x) \in \Omega: t \leqq T\}$ and $\Omega_{h}=\left\{(t, x) \in \Omega:(t, x)=(j k, v h), j, v_{i}\right.$ integers, $\left.k=\lambda h^{M}\right\}$. Further, for $U \in \mathfrak{M}_{h}$ we introduce the notation

$$
\begin{aligned}
\|U\|_{h, \Omega} & =\left(k h^{d} \sum_{X \in \Omega_{h}}|U(X)|^{2}\right)^{1 / 2}, \\
\|U\|_{h, r, \Omega, \Omega} & =\sum_{i \leq r,|\alpha| \leq s}\left\|\bar{\partial}_{t}^{i} \partial_{x}^{\alpha} U\right\|_{h, \Omega}, \\
\|U\|_{h, T} & =\|U\|_{h,\left(R^{1+d}\right)}, \quad\|U\|_{h, r, s, T}=\|U\|_{h, r, s,\left(R^{1+d}\right) r} .
\end{aligned}
$$

We now state the main theorem of this section.

THEOREM 3.1. Let $\Omega$ be a bounded domain in $R^{1+d}$ and let $L_{h}$, given by (2.2), be a parabolic (in the sense of $F$. John) difference operator in $\Omega$. Then, for any $\Omega_{1} \subset \subset$ $\Omega_{2} \subset \subset \Omega$ and any nonnegative integers $r, s$, there exist positive constants $C_{0}$ and $h_{0}$ such that for $T \in R$

$$
\begin{array}{r}
\|U\|_{h, r+1, s, \Omega_{1} r}+\|U\|_{h, r, M+s, \Omega_{1} r} \leqq C_{0}\left(\left\|L_{h} U\right\|_{h, r, s, \Omega_{2} r}+\|U\|_{h, \Omega_{2} r},\right. \\
h \leqq h_{0}, \quad U \in \mathbb{M}_{h} .
\end{array}
$$

Remark 3.1. It might seem more natural to use the norm

$$
\|U\|_{h, r, \Omega}^{*}=\sum_{M i+|\alpha| \leq r}\left\|\bar{\partial}_{t}^{i} \partial_{x}^{\alpha} U\right\|_{h, \Omega} .
$$

This norm is, however, unsuitable for the application in Section 4.

In order to be able to handle the implicit case, $A_{h} \neq I$, we define the fractional difference operators $\tilde{\partial}_{t}^{i}$ by

$$
\begin{aligned}
\tilde{\partial}_{t}^{i} & =I, \quad j=0, \\
& =h^{M-j} \bar{\partial}_{t}, \quad 1 \leqq j \leqq M .
\end{aligned}
$$

Note that $\tilde{\partial}_{t}^{M}=\bar{\partial}_{t}$. We often omit the subscript $t$.

The proof of Theorem 3.1 will be carried out in several steps. We first treat a principal part operator with constant coefficients and derive an estimate valid for $U \in \mathbb{D}_{h}(\Omega)$, where $\mathbb{D}_{h}(\Omega)=\left\{U \in \mathfrak{M}_{h}: U(X)=0\right.$ for $\left.X \notin \Omega\right\}$.

LEMMA 3.1. Let $\Omega \subset R^{1+d}$ be a bounded domain. Also, let $L_{h}^{0}$ be the principal part of a parabolic difference operator with coefficients independent of $X$. Then, there is a constant $C_{0}$ (which depends only on the constants in the definition of parabolicity, on the bound of the coefficients in $L_{h}^{0}$, and on the spatial diameter of $\Omega$ ) such that

$$
\sum_{i+|\alpha| \leqq M}\left\|\tilde{\partial}_{t}^{i} \partial_{x}^{\alpha} U\right\|_{h, T} \leqq C_{0}\left\|L_{h}^{0} U\right\|_{h, T}, \quad h \leqq 1, \quad U \in D_{h}(\Omega), \quad T \in R .
$$

Proof. Let $|\alpha|=M$. Set $T_{0}=\inf \{t:(t, x) \in \Omega\}$ and let $F$ and $H$ be the corresponding functions in Lemma 2.3. Then, using this lemma, the Parseval relation and Lemma 2.2, we get for $U \in D_{h}(\Omega)$,

$$
\left\|\partial_{x}^{\alpha} U\right\|_{n, T} \leqq\|H\|_{h}=(2 \pi)^{1 / 2(1+d)}\left\|\partial_{x}^{\alpha}(\cdot) L_{h}^{0}(\cdot, \cdot)^{-1} \hat{F}(\cdot, \cdot)\right\|_{L^{2}(\theta)} \leqq C\left\|L_{h}^{0} U\right\|_{h, T} .
$$

The discrete Poincaré inequality (cf. Lemma 2.4 in [11]) shows that

$$
\|U\|_{h, 0, M, T} \leqq C \max _{|\alpha|-M}\left\|\partial_{x}^{\alpha} U\right\|_{h, T}, \quad h \leqq 1, \quad U \in D_{h}(\Omega),
$$


where the constant $C$ depends only on the diameter of $\Omega$ in the $x$-space. Hence, we get

$$
\|U\|_{h, 0, M, T} \leqq C\left\|L_{h}^{0} U\right\|_{h, T}, \quad h \leqq 1, \quad U \in \mathbb{D}_{h}(\Omega) .
$$

Since $A_{h}^{0}$ has a bounded inverse on $l_{h}^{2}$, we conclude from (2.4) and (3.3) that

$$
\left\|\bar{\partial}_{\iota} U\right\|_{h, T} \leqq C\left(\left\|L_{h}^{0} U\right\|_{h, T}+\sum_{|\alpha|=M}\left\|\partial_{x}^{\alpha} U\right\|_{h, T}\right) \leqq C\left\|L_{h}^{0} U\right\|_{h, T} .
$$

If $j \neq 0$ and $j+|\alpha| \leqq M$, we thus have, using (3.2),

$$
\left\|\tilde{\partial}_{t}^{i} \partial_{x}^{\alpha} U\right\|_{h, T}=\left\|h^{M-i} \bar{\partial}_{t} \partial_{x}^{\alpha} U\right\|_{h, T} \leqq C\left\|\bar{\partial}_{t} U\right\|_{h, T} \leqq C\left\|L_{h}^{0} U\right\|_{h, T} .
$$

Together, (3.3) and (3.4) prove the lemma.

Our first step towards an inequality for an operator with variable coefficients is the following:

LEMMA 3.2. Let $L_{h}^{0}$ be the principal part of a difference operator which is parabolic in a bounded domain $\Omega \subset R^{1+d}$. Then, for any $\Omega_{1} \subset \subset \Omega$, there are positive constants $C_{0}, h_{0}$ and $\epsilon_{0}$ such that, if $h \leqq h_{0}$ and $T \in R$, we have

$$
\sum_{j+|\alpha| \leqq M}\left\|\tilde{\partial}_{t}^{j} \partial_{x}^{\alpha} U\right\|_{h, T} \leqq C_{0}\left\|L_{h}^{0} U\right\|_{h, T}
$$

for every function $U \in D_{h}\left(\Omega_{1}\right)$ whose support has diameter less than $\epsilon_{0}$.

Proof. Let $X_{0} \in \operatorname{supp} U$. Defining the operator $L_{h}^{0}\left(X_{0}\right)$ by

$$
L_{h}^{0}\left(X_{0}\right)=\sum_{\nu} a_{v}\left(X_{0}, 0\right) T^{\nu} \bar{\partial}-\sum_{|\alpha|=M, \nu} c_{\alpha \nu}\left(X_{0}, 0\right) T^{-1} T^{\nu} \partial^{\alpha},
$$

we have according to the preceding lemma with some constant $C_{1}$ independent of $X_{0}$

$$
\sum_{i+|\alpha| \leqq M}\left\|\tilde{\partial}^{i} \partial^{\alpha} U\right\|_{h, T} \leqq C_{1}\left\|L_{h}^{0}\left(X_{0}\right) U\right\|_{h, T}, \quad h \leqq 1, \quad U \in D_{h}\left(\Omega_{1}\right) .
$$

On the other hand, it is readily shown, using (2.4) and the regularity assumptions on $a_{v}$ and $c_{\alpha \nu}$, that for sufficiently small $h_{0}$ and $\epsilon_{0}$,

$$
\begin{aligned}
\left\|L_{h}^{0}\left(X_{0}\right) U-L_{h}^{0} U\right\|_{h, T} \leqq & \sum_{\nu}\left\|\left(a_{\nu}\left(X_{0}, 0\right)-a_{\nu}(X, 0)\right) T^{\nu} \bar{\partial} U\right\|_{h, T} \\
& +\sum_{|\alpha|=M, \nu}\left\|\left(c_{\alpha \nu}\left(X_{0}, 0\right)-c_{\alpha \nu}(X, 0)\right) T^{-1} T^{\nu} \partial^{\alpha} U\right\|_{h, T} \\
& \leqq \frac{1}{3 C_{1}}\left(\left\|\left.U\right|_{h 1,0, T}+\right\| U \|_{h, 0, M, T}\right),
\end{aligned}
$$

which evidently completes the proof.

We next extend this result to arbitrary functions in $D_{h}\left(\Omega_{1}\right)$.

LEMMA 3.3. Let $L_{h}$ be a difference operator which is parabolic in a bounded domain $\Omega \subset R^{1+d}$. Then, for any $\Omega_{1} \subset \subset \Omega$, there are positive constants $C_{0}$ and $h_{0}$ such that

$$
\begin{aligned}
\sum_{j+|\alpha| \leqq M}\left\|\tilde{\partial}_{t}^{i} \partial_{x}^{\alpha} U\right\|_{h, T} \leqq C_{0}\left(\left\|L_{h} U||_{h, T}+\right\| U \|_{h, 0, M-1, T-k}\right), & \\
& h \leqq h_{0}, \quad U \in \mathfrak{D}_{h}\left(\Omega_{1}\right), \quad T \in R .
\end{aligned}
$$

Proof. Let $\epsilon_{0}$ be the constant in Lemma 3.2. There exists a finite sequence $\left\{\phi_{i}\right\}_{i=1}^{K}$ of scalar valued functions in $C_{0}^{\infty}(\Omega)$ such that diam (supp $\left.\phi_{i}\right)<\epsilon_{0}$ and $\sum \phi_{i}(X)=1$ in $\Omega_{1}$. Now, let $U \in D_{h}\left(\Omega_{1}\right)$. Then we have $U=\sum \phi_{i} U$ and, using Lemma 3.2 on $\phi_{i} U$, we get for $j+|\alpha| \leqq M$ 


$$
\left\|\tilde{\partial}^{i} \partial^{\alpha} U\right\|_{h, T} \leqq \sum_{i}\left\|\tilde{\partial}^{i} \partial^{\alpha}\left(\phi_{i} U\right)\right\|_{h, T} \leqq C \sum_{i}\left\|L_{h}^{0}\left(\phi_{i} U\right)\right\|_{h, T} .
$$

Since the $\phi_{i}$ are uniformly bounded, we have therefore

$$
\begin{aligned}
& \sum_{j+|\alpha| \leq M}\left\|\tilde{\partial}^{i} \partial^{\alpha} U\right\|_{h, T} \\
& \leqq C_{1}\left(\left\|L_{h} U\right\|_{h, T}+\left\|L_{h}^{0} U-L_{h} U\right\|_{h, T}+\max _{i}\left\|L_{h}^{0} \phi_{i} U-\phi_{i} L_{h}^{0} U\right\|_{h, T}\right) .
\end{aligned}
$$

The last two terms inside the parentheses are estimated for sufficiently small $h$ by use of Lemma 2.1, (2.4), and the Leibniz rule:

$$
\begin{array}{r}
\left\|L_{h}^{0} U-L_{h} U\right\|_{h, T} \leqq \frac{1}{4 C_{1}}\|U\|_{h, 1,0, T}+C\|U\|_{h, 0, M-1, T-k}, \\
\left\|L_{h}^{0} \phi_{i} U-\phi_{i} L_{h}^{0} U\right\|_{h, T} \\
\leqq \frac{1}{4 C_{1}}\left[\|U\|_{h, 1,0, T}+\|U\|_{h, 0 . M, T}\right]+C\|U\|_{h, 0, M-1, T-k} .
\end{array}
$$

The estimates (3.5)-(3.7) prove the lemma.

To prove Theorem 3.1, we have to reduce the subscript $M-1$ in Lemma 3.3 to zero. This can be done using a discrete analogue of a norm used by Friedrichs in his investigations on elliptic differential equations (cf. [2] and [11]). Let $\Omega$ be a bounded domain and let $\Omega_{1} \subset \subset \Omega_{2} \subset \subset \Omega$. Take a scalar valued and nonnegative function $\phi \in C_{0}^{\infty}\left(\Omega_{2}\right)$ with $\phi=1$ in $\Omega_{1}$. We introduce the norms

$$
\|U\|_{h, s, T}^{(\phi)}=\sum_{m+|\alpha| \leq s}\left\|\phi^{m+|\alpha|} \tilde{\partial}_{t}^{m} \partial_{x}^{\alpha} U\right\|_{h, \Omega_{2} T}, \quad 0 \leqq s \leqq M .
$$

We now give a sequence of three lemmas concerning these norms. For simplicity, we shall write $T^{i, \mu}=T_{t}^{i} T_{x}^{\mu}$ and $\partial^{i, \alpha}=\partial_{t}^{i} \partial_{x}^{\alpha}$.

Lemma 3.4. Given $i, \mu, j, \beta, q, m, \alpha, s$ with $q \geqq 1, s \geqq 0$ and $j+|\beta|+m+$ $|\alpha| \leqq q, m+|\alpha| \leqq s \leqq M$, there are positive constants $C_{0}$ and $h_{0}$ such that

$$
\left\|\left(T^{i, \mu} \partial^{j, \beta} \phi^{q}\right) \tilde{\partial}_{t}^{m} \partial_{x}^{\alpha} U\right\|_{h, T} \leqq C_{0}\|U\|_{h, s, T}^{(\phi)}, \quad h \leqq h_{0}, \quad U \in \mathscr{T}_{h}, \quad T \in R .
$$

Proof. If $m+|\alpha|=0,(3.8)$ holds trivially. In particular, the statement is true for $s=0$. Let $\psi=T^{i, \mu} \partial^{i, \beta} \phi^{q}$. If $j+|\beta|+m+|\alpha| \leqq q$, we have, by a Taylor expansion,

$$
|\psi| \leqq C \sum_{r=0}^{m+|\alpha|} h^{r} \phi^{m+|\alpha|-r} .
$$

For small $h, \psi=0$ outside some $\Omega_{3} \subset \subset \Omega_{2}$ and we obtain for $0<m+|\alpha| \leqq s$

$\left\|\psi \tilde{\partial}^{m} \partial^{\alpha} U\right\|_{h, T}$

$$
\begin{aligned}
& =\left\|\psi \tilde{\partial}^{m} \partial^{\alpha} U\right\|_{h, \Omega_{\mathrm{s}} r} \leqq C \sum_{r=0}^{m+|\alpha|}\left\|\phi^{m+|\alpha|-r} h^{r} \tilde{\partial}^{m} \partial^{\alpha} U\right\|_{h, \Omega_{\mathrm{s}} T} \\
& \leqq C\left[\|U\|_{h, s, T}^{(\phi)}+\sum_{r=1}^{m+|\alpha|} \sum_{|\gamma|+n-m+|\alpha|-r ; l, \nu}\left\|\left(T^{l, \eta} \phi^{m+|\alpha|-r}\right) \tilde{\partial}^{n} \partial^{\gamma} U\right\|_{h, \Omega_{3} r}\right] .
\end{aligned}
$$

Now, the statement follows from (3.9) successively for $s=1, \cdots, M$. 
LEMmA 3.5. Given $m, \alpha, q$, and $s$ with $1 \leqq s \leqq q$ and $m+|\alpha| \leqq s \leqq M$, there are positive constants $C_{0}$ and $h_{0}$ such that for $h \leqq h_{0}, U \in \mathfrak{T T}_{h}$ and $T \in R$,

$$
\begin{gathered}
\left\|\tilde{\partial}_{t}^{m} \partial_{x}^{\alpha}\left(\phi^{q} U\right)-\phi^{q} \tilde{\partial}_{t}^{m} \partial_{x}^{\alpha} U\right\|_{h, T} \leqq C_{0}\|U\|_{h, s-1, T}^{(\phi)}, \\
\left\|\tilde{\partial}_{t}^{m} \partial_{x}^{\alpha}\left(\phi^{\alpha} U\right)\right\|_{h, T} \leqq C_{0}\|U\|_{h, s, T}^{(\phi)} .
\end{gathered}
$$

Proof. The inequality (3.11) clearly follows from (3.10), which we prove separately for the cases $m \neq 0$ and $m=0$. In the first case we get, using the Leibniz rule,

$$
\begin{aligned}
& \tilde{\partial}^{m} \partial^{\alpha}\left(\phi^{q} U\right)-\phi^{a} \tilde{\partial}^{m} \partial^{\alpha} U \\
&=\sum_{0<\beta \leq \alpha}\left(\begin{array}{l}
\alpha \\
\beta
\end{array}\right)\left(\partial^{\beta} \phi^{q}\right)\left(T^{\beta} \tilde{\partial}^{m} \partial^{\alpha-\beta} U\right)+\sum_{0 \leqq \beta \leq \alpha}\left(\begin{array}{l}
\alpha \\
\beta
\end{array}\right)\left(\tilde{\partial}^{m} \partial^{\beta} \phi^{q}\right)\left(T^{-1, \beta} \partial^{\alpha-\beta} U\right),
\end{aligned}
$$

and so (3.10) follows from (3.8). If $m=0$ the second sum does not appear and (3.10) follows for this case too.

The last lemma is of interpolation type.

LEMmA 3.6. Given $s, 1 \leqq s \leqq M$, and $\epsilon>0$ there are positive constants $C_{n}$ ' and $h_{0}$ such that

$$
\|U\|_{h, s-1, T}^{(\phi)} \leqq \epsilon\|U\|_{h, s, T}^{(\phi)}+C_{0}\|U\|_{h, 0, T}^{(\phi)}, \quad h \leqq h_{0}, \quad U \in 9 \Re_{h}, \quad T \in R .
$$

Proof. We prove the lemma by induction on $s \leqq M$. If $s=1$ the inequality (3.12) is obvious. Now suppose that (3.12) holds for $s=1, \cdots, S-1$. To establish it for $s=S \geqq 2$, we have to estimate only those terms $\left\|\phi^{m+|\alpha|} \tilde{\partial}^{m} \partial^{\alpha} U\right\|_{h, T}$ for which $m+|\alpha|=S-1$. This will be done first for $m \neq 0$ and then for $m=0$. We use the notation $(U, V)_{h, T}=\sum_{t \leqq T} \sum_{i=1}^{N} u_{i}(X) \bar{v}_{i}(X)$. If $m \neq 0$, we get

$$
\begin{array}{rlrl}
\left\|\phi^{S-1} \tilde{\partial}^{m} \partial^{\alpha} U\right\|_{h, T}^{2} & =\left(\phi^{S} \tilde{\partial}^{m} \partial^{\alpha} U, \phi^{S-2} \tilde{\partial}^{m} \partial^{\alpha} U\right)_{h, T} & \\
& =\left(\phi^{S} \tilde{\partial}^{m+1} \partial^{\alpha} U, \phi^{S-2} \tilde{\partial}^{m-1} \partial^{\alpha} U\right)_{h, T}, & & m \geqq 2, \\
& =\left(\phi^{S} \tilde{\partial}^{2} \partial^{\alpha} U, \phi^{S-2} \partial^{\alpha}\left(U-T_{t}^{-1} U\right)\right)_{h, T}, & & m=1,
\end{array}
$$

and then, using the Schwarz inequality and (3.8), we find that

$$
\left\|\phi^{S-1} \tilde{\partial}^{m} \partial^{\alpha} U\right\|_{h, T}^{2} \leqq C\|U\|_{h, S, T}^{(\phi)} \cdot\|U\|_{h, S-2, T}^{(\phi)} .
$$

If $m=0$, it follows from the argument in the corresponding proof in [11] that (3.13) holds. From (3.13) and the induction assumption, we may conclude

$$
\begin{aligned}
\|U\|_{h, S-1, T}^{(\phi)} & \leqq \frac{\epsilon}{2}\|U\|_{h, S, T}^{(\phi)}+C_{\epsilon}\|U\|_{h, S-2, T}^{(\phi)} \\
& \leqq \frac{\epsilon}{2}\|U\|_{h, S, T}^{(\phi)}+\frac{1}{2}\|U\|_{h, S-1, T}^{(\phi)}+\frac{1}{2} C_{0}\|U\|_{h, 0, T}^{(\phi)}
\end{aligned}
$$

This proves the lemma.

It is now possible for us to prove Theorem 3.1 .

Proof of Theorem 3.1. We first prove the theorem for the case $r=s=0$. We assume that $h$ is small so that the lemmas apply. We have

$$
\begin{gathered}
\|U\|_{h, 1,0, \Omega_{1} T}+\|U\|_{h, 0, M, \Omega_{1} T} \leqq\|U\|_{h, M, T}^{(\phi)} \\
\|U\|_{h, M, T}^{(\phi)}=\sum_{m+|\alpha|=M}\left\|\phi^{M} \tilde{\partial}^{m} \partial^{\alpha} U\right\|_{h, \Omega_{2} T}+\|U\|_{h, M-1, T}^{(\phi)}
\end{gathered}
$$


Application of (3.10) in (3.15) gives

$$
\|U\|_{h, M, T}^{(\phi)} \leqq \sum_{m+|\alpha|-M}\left\|\tilde{\partial}^{m} \partial^{\alpha}\left(\phi^{M} U\right)\right\|_{h, T}+C\|U\|_{h, M-1, T}^{(\phi)} .
$$

Using Lemma 3.3 on the terms in the sum, we see that

$$
\|U\|_{h, M, T}^{(\phi)} \leqq C\left[\left\|L_{h}\left(\phi^{M} U\right)\right\|_{h, T}+\left\|\phi^{M} U\right\|_{h, 0, M-1, T}+\|U\|_{h, M-1, T}^{(\phi)}\right] .
$$

By (3.11), the following holds:

$$
\left\|\phi^{M} U\right\|_{h, 0, M-1, T} \leqq C\|U\|_{h, M-1, T}^{(\phi)} \text {. }
$$

To permit the switching of $L_{h}$ and $\phi^{M}$ in (3.16), we show that

$$
\left\|L_{h}\left(\phi^{M} U\right)-\phi^{M} L_{h} U\right\|_{h, T} \leqq C\|U\|_{h, M-1, T}^{(\phi)} .
$$

To do this, we first notice from Lemma 2.1 that

$$
\begin{aligned}
L_{h}\left(\phi^{M} U\right)-\phi^{M} L_{h} U= & \sum_{\nu} a_{\nu}\left[T^{\nu} \bar{\partial}\left(\phi^{M} U\right)-\phi^{M} T^{\nu} \bar{\partial} U\right] \\
& -\sum_{|\alpha| \leqslant M, \nu} c_{\alpha \nu}\left[T^{-1} T^{\nu} \partial^{\alpha}\left(\phi^{M} U\right)-\phi^{M} T^{-1} T^{\nu} \partial^{\alpha} U\right]=P+Q .
\end{aligned}
$$

We infer from the Leibniz rule and Lemma 3.4 that

$$
\begin{aligned}
\|P\|_{h, T} & =\left\|\sum_{\nu} a_{\nu}\left(T^{\nu} \phi^{M}-\phi^{M}\right) T^{\nu} \bar{\partial} U+a_{\nu} T^{\nu}\left[\left(\bar{\partial} \phi^{M}\right) T^{-1} U\right]\right\|_{h, T} \\
& \leqq C \sum_{|\beta|=1, \nu}\left\|\left(T^{\nu} \partial^{\beta} \phi^{M}\right) h \bar{\partial} U\right\|_{h, T}+C\|U\|_{h, 0, T}^{(\phi)} \leqq C\|U\|_{h, M-1, T}^{(\phi)} .
\end{aligned}
$$

The second term, $Q$, is estimated in a similar way and therefore (3.18) follows. Indeed, our use of the operators $\tilde{\partial}_{t}^{m}$ was necessary for the estimation of $\left\|\left(T^{\nu} \partial^{\beta} \phi^{M}\right) h \bar{\partial} U\right\|_{h, T}=$ $\left\|\left(T^{\nu} \partial^{\beta} \phi^{M}\right) \tilde{\partial}^{M-1} U\right\|_{h, T}$ in the inequality for $P$. In the explicit case, $A_{h}=I$, the first part of $P$ would disappear and we could do without the operators $\tilde{\partial}_{t}^{m}$. Using (3.17), (3.18), and Lemma 3.6, we transform (3.16) into

$$
\|U\|_{h, M, T}^{(\phi)} \leqq C\left(\left\|L_{h} U\right\|_{h, \Omega_{2} T}+\|U\|_{h, \Omega_{2} T}\right)
$$

Thus, by (3.14), we have proved (3.1) for $r=s=0$. By induction on $s$, we deduce after some computation

$$
\|U\|_{h, 1, s, \Omega_{1} T}+\|U\|_{h, 0, M+s, \Omega_{1} T} \leqq C\left(\left\|L_{h} U\right\|_{h, 0, s, \Omega_{2} T}+\|U\|_{h, \Omega_{2} T}\right),
$$

which is the statement for $r=0$. Finally, (3.1) follows for arbitrary $r, s$ from (3.19) by induction on $r$.

4. Convergence of Difference Quotients. Now, consider in a bounded domain $\Omega \subset R^{1+d}$ the equation

$$
L U=F,
$$

where $L$ is a parabolic differential operator and $F \in C^{\infty}(\Omega)$ is a given $N$-vector valued function. It is then known that any solution belongs to $C^{\infty}(\Omega)$ (see e.g. [1]). For each value of the positive parameter $h$, we introduce a mesh $\left\{X \in R^{1+d}: X=(j k, \nu h)\right.$, $j, \nu_{i}$ integers, $\left.k=\lambda h^{M}\right\}$, where $M$ is the order of the operator $L$, and denote by 
$\Omega_{h}$, as before, the set of meshpoints in $\Omega$. Let $L_{h}$ be a parabolic difference operator consistent with $L$ in $\Omega$. Further, let $M_{h}$ be an operator of the form

$$
\begin{aligned}
& M_{h} U(t, x)=\sum_{j=1}^{J} \sum_{|\nu| \leqq K} m_{i, \nu}(t, x, h) U\left(t-\theta_{i} k, x+\nu h\right), \\
& \quad 0 \leqq \theta_{i} \leqq 1, \quad m_{j, \nu} \in C_{N}^{\infty}(\Omega, h) .
\end{aligned}
$$

This operator is required to be consistent with the identity operator. We then approximate $(4.1)$ by the difference equation

$$
L_{h} U_{h}=M_{h} F \quad \text { in } \Omega_{h}^{\circ},
$$

where $\Omega_{h}^{o}$ is a subset of $\Omega_{h}$ such that for any $\Omega_{1} \subset \subset \Omega$, we have $\Omega_{1, h} \subset \Omega_{h}^{o}$ for $h$ small enough, and such that $L_{h} U$ and $M_{h} U$ are determined in $\Omega_{h}^{\circ}$ by the values of $U$ in $\Omega$. Any function $U_{h}$ defined in $\Omega_{h}$ and satisfying (4.2) is called a solution of (4.2). We say that (4.2) approximates (4.1) with order of accuracy $p$ if for any $U \in C^{\infty}(\Omega)$ and any $X \in \Omega$,

$$
L_{h} U(X)-M_{h} L U(X)=O\left(h^{p}\right) \text { as } h \rightarrow 0 .
$$

We observe from (4.2) that the operator $M_{h}$ has no effect on our problem if $F=0$. In fact, operators of this type were introduced to get the same rate of convergence for the Cauchy problem for certain inhomogeneous differential equations as for the corresponding homogeneous ones (cf. [3]).

We shall consider scalar differential operators $Q$ and difference operators $Q_{h}$ which for simplicity are assumed to be of the form

$$
Q=\sum_{|\alpha| \leqq Q} Q_{\alpha}(t, x) D_{x}^{\alpha}, \quad Q_{\alpha} \in C^{\infty}(\Omega),
$$

and

$$
Q_{h}=\sum_{|\alpha| \leqq Q, \nu} q_{\alpha \nu}(t, x, h) T_{x}^{\nu} \partial_{x}^{\alpha}, \quad q_{\alpha \nu} \in C_{1}^{\infty}(\Omega, h),
$$

respectively. We say that $Q_{h}$ approximates $Q$ with order of accuracy $p$ if for any $U \in C^{\infty}(\Omega)$ and any $X \in \Omega$,

$$
Q_{h} U(X)-Q U(X)=O\left(h^{p}\right) \text { as } h \rightarrow 0 .
$$

We also introduce the maximum norm

$$
|U|_{h, \Omega}=\max _{x \in \Omega_{h}}|U(X)| .
$$

In this section, we shall prove the following convergence theorem as an application of the a priori estimate derived in Section 3.

THEOREM 4.1. Let $\Omega$ be a bounded domain in $R^{1+d}$. Further, let the difference operator $L_{k}$ in (2.2) and the differential operator $L$ in (2.1) be parabolic in $\Omega$ in the sense of $F$. John and Petrouskii, respectively. Assume that (4.2) approximates (4.1) with order of accuracy $p$ and that $Q_{h}$, given by (4.4), approximates $Q$, given by (4.3), with the same order of accuracy. Then, if $U$ is a solution of (4.1) and $\Omega_{1} \subset \subset \Omega_{2} \subset \subset \Omega$, there exist positive constants $C_{0}$ and $h_{0}$ such that

$$
\left|Q_{h} U_{h}-Q U\right|_{h, \Omega_{2} T} \leqq C_{0}\left(h^{p}+\| U_{h}-\left.U\right|_{h, \Omega_{2} T}\right), \quad h \leqq h_{0}, \quad T \in R,
$$

for any solution $U_{h}$ of (4.2).

From this theorem, we conclude that if for any $\Omega_{2} \subset \subset \Omega$ we have $\left\|U_{h}-U\right\|_{h, \Omega_{2}}=$ $O\left(h^{r}\right)$ as $h \rightarrow 0$, then for any $\Omega_{1} \subset \subset \Omega$, 


$$
\left|Q_{h} U_{h}-Q U\right|_{h, \Omega_{1}}=O\left(h^{\min (p, r)}\right) \quad \text { as } h \rightarrow 0 .
$$

In particular, choosing $Q_{k}=Q=I$, we see that convergence in the norm $\|\cdot\|_{h, \Omega_{z}}$ implies uniform convergence in $\Omega_{1}$.

In order to prove the theorem, we need a result due to Sobolev [9]. To state this we introduce additional notation. Let $\omega$ be a bounded set in $R^{d}$. For functions $V$ defined on the cubic mesh $\left\{x \in R^{d}: x=\nu h\right\}$, we set

$$
\begin{aligned}
\|V\|_{h, \omega} & =\left(h^{d} \sum_{\nu h \in \omega}|V(\nu h)|^{2}\right)^{1 / 2}, \\
\|V\|_{h, s, \omega} & =\sum_{|\alpha| \leqq s}\left\|\partial_{x}^{\alpha} V\right\|_{h, \omega}, \\
|V|_{h, \omega} & =\max _{\nu h \in \omega}|V(\nu h)| .
\end{aligned}
$$

The Sobolev Inequality. For any two domains $\omega_{1} \subset \subset \omega_{2} \subset \subset R^{d}$, there exists a constant $C$ such that

$$
|V|_{h, \omega_{1}} \leqq C \| V||_{h,\{d / 2\}+1, \omega_{2}},
$$

where $[d / 2]$ denotes the largest integer $\leqq d / 2$.

The following lemma contains an inequality related to the Sobolev inequality.

LEMMA 4.1. Let $\Omega_{1}$ and $\Omega_{3}$ be open cylinders, $\Omega_{i}=\left(T_{1}, T_{2}\right) \times \omega_{i}, i=1,3$, where $\omega_{1}$ and $\omega_{3}$ are bounded domains in $R^{d}$ such that $\omega_{1} \subset \subset \omega_{3}$. Then, if $T-T_{1}$ is bounded below by some positive number, there is a constant $C_{0}$ such that

$$
|U|_{h, \Omega_{1}} \leqq_{0}\|U\|_{h, 1,\{d / 2]+1, \Omega_{3} T,}, \quad U \in \mathfrak{T}_{h} .
$$

Proof. The proof will consist of two parts, each of which is an application of the Sobolev inequality. First, we note that, according to that inequality, there is a constant $C$ such that for any $t=j k$,

$$
\max _{\nu h \in \omega_{1}}|U(t, \nu h)| \leqq C\|U(t, \cdot)\|_{h,\{d / 2\}+1, \omega_{s},} \quad U \in \mathbb{N}_{h} .
$$

Hence, (4.5) would follow in the case $T<T_{2}$ if we could prove that

$$
\begin{aligned}
& g(t) \equiv\|U(t, \cdot)\|_{h,[d / 2]+1, \omega_{s}} \leqq C\|U\|_{h, 1,[d / 2]+1, \Omega_{3} T} \\
& \qquad U \in \mathscr{N}_{h}, \quad T_{1}<t=j k \leqq T<T_{2} .
\end{aligned}
$$

In the case $T \geqq T_{2}$, which is treated similarly, we must prove this inequality instead for $T_{1}<t=j k<T_{2}$. The Sobolev inequality for $d=1$ shows that

$$
g(j k) \leqq C\left(\|g\|_{k,\left(T_{1}, T\right\}}+\left\|\bar{\partial}_{t} g\right\|_{k,\left(T_{1}, T\right\}}\right), \quad T_{1}<j k \leqq T,
$$

where the constant $C$ depends only on the difference $T-T_{1}$. It now remains to substitute the expression for $g$ into the right member of (4.7). Let the integers $R$ and $S$ be such that $(R-1) k \leqq T_{1}<R k$ and $S k \leqq T<(S+1) k$. We then have

$$
\begin{aligned}
& \|g\|_{k,\left(T_{1}, T\right]}^{2}=\sum_{i=R}^{S} k|g(i k)|^{2} \leqq C\|U\|_{h, 0,\{d / 2\}+1, \Omega_{3} T}^{2}, \\
& \left\|\bar{\partial}_{t} g\right\|_{k,\left(T_{1}, T\right\}}^{2} \\
& =\sum_{i=R}^{S} k^{-1}\left[\sum_{|\alpha| \leq\{d / 2\}+1}\left\|\partial_{x}^{\alpha} U(i k, \cdot)\right\|_{h, \omega_{3}}-\left\|\partial_{x}^{\alpha} U((i-1) k, \cdot)\right\|_{h, \omega_{3}}\right]^{2} \\
& \leqq C\|U\|_{h, 1,\{d / 2\}+1, \Omega_{3} r}^{2},
\end{aligned}
$$


where the constants are independent of $T$. Together (4.7)-(4.9) prove (4.6). This completes the proof.

Remark 4.1. Direct application of the $(d+1)$-dimensional Sobolev inequality on the pair $\left(\Omega_{1}, \Omega_{3}\right)$ in Lemma 4.1 would give an estimate similar to (4.5). The righthand side of this, however, would depend on difference quotients in the $t$-direction of order greater than 1.

Now we can prove Theorem 4.1.

Proof of Theorem 4.1. It is evidently enough to prove the theorem for the case $\Omega_{1}=\left(T_{1}, T_{2}\right) \times \omega_{1}$ with $\omega_{1}$ a domain in $R^{d}$ and $\Omega_{1} \subset \subset \Omega_{2}$. Also, we may assume that $T-T_{1}$ is bounded below by some positive number. We choose the domain $\Omega_{4}$ such that $\Omega_{2} \subset \subset \Omega_{4} \subset \subset \Omega$ and let $h$ be small so that $\Omega_{4, h} \subset \Omega_{h}^{o}$. We then extend $U_{h}$ in an arbitrary way to be a function in $\mathfrak{T}_{h}$. The triangle inequality gives

$$
\left|Q_{h} U_{h}-Q U\right|_{h, \Omega_{2} T} \leqq\left|Q_{h}\left(U_{h}-U\right)\right|_{h, \Omega_{1} T}+\left|\left(Q_{h}-Q\right) U\right|_{h, \Omega_{1} T} .
$$

Since $Q_{h}$ approximates $Q$ with order of accuracy $p$ and $U \in C^{\infty}(\Omega)$, we have for the second term

$$
\left|\left(Q_{h}-Q\right) U\right|_{h, \Omega_{2} T} \leqq C h^{p}
$$

if $h$ is sufficiently small. To estimate the first term, we let $\Omega_{3}=\left(T_{1}, T_{2}\right) \times \omega_{3}$ be a cylinder with $\omega_{1} \subset \subset \omega_{3}$ and $\Omega_{3} \subset \subset \Omega_{2}$. By Lemma 4.1 and Theorem 3.1, we obtain

$$
\begin{aligned}
\left|Q_{h}\left(U_{h}-U\right)\right|_{h, \Omega_{1} T} & \leqq C\left\|U_{h}-U\right\|_{h, 1,\{d / 2]+1+a, \Omega_{3} T} \\
& \leqq C\left[\left\|L_{h}\left(U_{h}-U\right)\right\|_{h, 0,\{d / 2]+1+a, \Omega_{2} T}+\left\|U_{h}-U\right\|_{\left.h, \Omega_{2} T\right]}\right.
\end{aligned}
$$

In $\Omega_{4, h}$, we have $L_{h}\left(U_{h}-U\right)=M_{h} F-L_{h} U=\left(M_{h} L-L_{h}\right) U$. Making a Taylor expansion and using the assumption about the order of accuracy, we find for $X \in$ $\Omega_{4}$ and small $h$,

$$
\left(M_{h} L-L_{h}\right) U(X)=\sum_{i=p}^{p+r-1} h^{i} U_{i}(X)+h^{p+r} U_{p+r}(X, h),
$$

$$
r=[d / 2]+1+q,
$$

where $U_{i}(X)$ is a linear combination of certain derivatives of $U$ at the point $X$ for $i \leqq p+r-1$ and $U_{p+r}(X, h)$ is a linear combination of certain derivatives of $U$ at the points $(t-\theta(X, h) k, x+\nu(X, h) h)$, where $(t, x)=X$ and $0 \leqq \theta \leqq 1$. Since $U \in C^{\infty}(\Omega)$, we thus get, for small $h$,

$$
\left\|L_{h}\left(U_{h}-U\right)\right\|_{h, 0,\{d / 2]+1+q, \Omega_{2} T} \leqq C h^{p}, \quad T \in R .
$$

(4.10)-(4.12) and (4.14) prove the theorem.

Remark 4.2. It is seen from the proofs of Theorem 3.1 and Theorem 4.1 that the regularity assumptions in Section 2 can be considerably relaxed. For the proof of Theorem 4.1 it suffices to know about the solution $U$ that $U \in C^{p+r+M}(\Omega)$, $r=[d / 2]+1+q$. If, however, we had used the norm proposed in Remark 3.1 or the idea in Remark 4.1, we would have to require more than this since $k=\lambda h^{M}$ (cf. (4.13)).

5. Examples. We now give two examples with $d=1$ and $N=1$ to illustrate our results. In these examples $L$ is the heat operator, 


$$
L=\frac{\partial}{\partial t}-\frac{\partial^{2}}{\partial x^{2}}
$$

and $L_{h}$ is the parabolic difference operator,

$$
L_{h}=\bar{\partial}_{t}-T_{t}^{-1} T_{x}^{-1} \partial_{x}^{2}, \quad k=\lambda h^{2}, \quad \lambda<\frac{1}{2} .
$$

Example 5.1. We consider the problem

$$
\begin{aligned}
L U & =F \quad \text { in } \Omega=\{(t, x): 0<t \leqq T, 0<x<1\}, \\
U & =f \quad \text { on } \Gamma=\bar{\Omega} \backslash \Omega,
\end{aligned}
$$

where we assume that $F \in C^{\infty}(\Omega)$ and $f$ are such that the problem admits a unique solution $U$ with $D_{i}^{i} D_{x}^{i} U \in C(\bar{\Omega})$ for $2 i+j \leqq 6$. For the numerical solution of this problem, we use the scheme

$$
\begin{gathered}
L_{h} U_{h}=M_{h} F \quad \text { in } \Omega_{h}, \\
U_{h}=f \quad \text { on } \Gamma_{h}, \quad h=1 / n, \quad \lambda=\frac{1}{6},
\end{gathered}
$$

where

$$
M_{h} F(t, x)=\frac{1}{3} F(t, x)+\frac{1}{12}[F(t, x+h)+F(t, x-h)]+\frac{1}{2} F(t-k, x) .
$$

The use of an operator $M_{h} \neq I$ is justified by the fact that for any smooth function $V$, we have $\left(L_{h}-M_{h} L\right) V=O\left(h^{4}\right)$ as $h \rightarrow 0$ while only $\left(L_{h}-L\right) V=O\left(h^{2}\right)$ as $h \rightarrow$ 0 . Let $U_{h}$ be the solution of the scheme. From the definition of $L_{h}$ we derive easily that

$$
|V|_{h, \bar{\Omega}} \leqq|V|_{h, \Gamma}+C\left|L_{h} V\right|_{h, \Omega}
$$

for any mesh-function $V$. Replacing $V$ by $U_{h}-U$, we thus have

$$
\left|U_{h}-U\right|_{h, \tilde{\Omega}} \leqq C\left|L_{h}\left(U_{h}-U\right)\right|_{h, \Omega}=C\left|\left(M_{h} L-L_{h}\right) U\right|_{h, \Omega}=O\left(h^{4}\right) \text { as } h \rightarrow 0,
$$

and consequently also $\left\|U_{h}-U\right\|_{h, \Omega}=O\left(h^{4}\right)$ as $h \rightarrow 0$. Hence, if $Q_{h}$ approximates $Q$ with order of accuracy 4 and $\Omega_{1} \subset \subset\{(t, x): 0<t<T, 0<x<1\}$, Theorem 4.1 gives

$$
\left|Q_{h} U_{h}-Q U\right|_{h, \Omega_{1}}=O\left(h^{4}\right) \quad \text { as } h \rightarrow 0 .
$$

Example 5.2. We consider the problem

$$
\begin{aligned}
L U & =0 \quad \text { in } \Omega=\{(t, x): 0<t, 0<x<1\}, \\
U(0, x) & =f(x), \quad 0 \leqq x \leqq 1, \\
U(t, 0) & =U(t, 1)=0, \quad 0<t,
\end{aligned}
$$

where we assume that the function $f$ is continuous and $f(0)=f(1)=0$. The corresponding discrete problem will be

$$
\begin{aligned}
L_{h} U_{h} & =0 \text { in } \Omega_{h}, \\
U_{h}(0, x) & =f(x), \quad 0 \leqq x=j h \leqq 1, \\
U_{h}(t, 0) & =U(t, 1)=0, \quad t=i k>0,
\end{aligned}
$$


where $h$ is the reciprocal of some positive integer. Let $U$ be the solution of (5.1) and $U_{h}$ be the solution of (5.2). By extending our mixed problems to pure initialvalue problems, we see from [12] (cf. [5], [7], and [8]) that

$$
\left|U_{h}-U\right|_{h, \Omega}=O\left(h^{r}\right) \quad \text { as } h \rightarrow 0,
$$

where

$$
\begin{aligned}
r & =\alpha & & \text { if } f \in \operatorname{Lip}_{\alpha}[0,1], \\
& =1+\alpha & \text { if } f^{\prime} \in \operatorname{Lip}_{\alpha}[0,1], & 0<\alpha \leqq 1 .
\end{aligned}
$$

Hence, if $Q_{h}$ approximates $Q$ sufficiently well and $\Omega_{1}$ is a bounded domain such that $\bar{\Omega}_{1} \subset \Omega$, we get from Theorem 4.1 ,

$$
\left|Q_{h} U_{h}-Q U\right|_{h, \Omega_{1}}=O\left(h^{r}\right) \quad \text { as } h \rightarrow 0 .
$$

In case $f$ is only continuous, $O\left(h^{r}\right)$ should be replaced by $o(1)$ above. These results could be obtained directly from [12]. Replacing $L_{h}$ in (5.2) by the implicit CrankNicolson operator $\tilde{L}_{h}$,

$$
\tilde{L}_{h}=\bar{\partial}_{t}-\frac{1}{2}\left[I+T_{t}^{-1}\right] T_{x}^{-1} \partial_{x}^{2}, \quad k=\lambda h^{2},
$$

which is parabolic for any fixed $\lambda, 0<\lambda<\infty$, we deduce easily analogous results (cf. also [6]).

Department of Mathematics

Chalmers Institute of Technology and

The University of Göteborg

Göteborg, Sweden

1. A. Friedman, Partial Differential Equations of Parabolic Type, Prentice-Hall, Englewood Cliffs, N. J., 1964. MR 31 \#6062.

2. K. O. FrIEDRICHS, "On the differentiability of solutions of linear elliptic differential equations," Comm. Pure Appl. Math, v. 6, 1953, pp. 299-326. MR 15, 430.

3. S. HÅRANGÅRD, "Some results on the rate of convergence for discrete initial-value problems," Report, Department of Mathematics, Chalmers Institute of Technology and the University of Goteborg.

4. F. JoHN, "On integration of parabolic equations by difference methods," Comm. Pure Appl. Math., v. 5, 1952, pp. 155-211. MR 13, 947.

5. M. L. JUNCOSA \& D. M. Young, "On the order of convergence of the solution of a difference equation to a solution of the diffusion equation," J. Soc. Indust. Appl. Math., v. 1, 1953, pp. 111-135. MR 15, 746.

6. M. L. Juncosa \& D. M. Young, "On the Crank-Nicolson procedure for solving parabolic partial differential equations," Proc. Cambridge Philos. Soc., v. 53, 1957, pp. 448461. MR 19, 583.

7. J. LÖFSTRÖM, "Besov spaces in the theory of approximation," Ann. Mat. Pura Appl., (4), v. 85,1970 , pp. $93-184$.

8. J. PeETRE \& V. ThOMÉE, "On the rate of convergence for discrete initial-value problems," Math. Scand., v. 21, 1967, pp. 159-176.

9. S. L. SoBolev, "On estimates for certain sums for functions defined on a grid," Izv. Akad. Nauk SSSR Ser. Mat., v. 4, 1940, pp. 5-16. (Russian) MR 1, 298.

10. V. THомée, "Parabolic difference operators," Math. Scand., v. 19, 1966, pp. 77-107. MR 35 \#590.

11. V. THOMÉE \& B. WESTERGReN, "Elliptic difference equations and interior regularity," Numer. Math., v. 11, 1968, pp. 196-210. MR 36 \#7347.

12. O. WIDLUND, "On the rate of convergence for parabolic difference schemes. II," Comm. Pure Appl. Math., v. 23, 1970, pp. 79-96. 